\title{
Duration of Serum Phosphorus Control Associated with Overall Mortality in Patients Undergoing Peritoneal Dialysis
}

\author{
Nirong Gong Zhiwen Xiao Fen Zhang Xiaohong Zhong Yanfang He \\ Zhixiu Yi Dan Tang Cong Yang Yanhong Lin Jing Nie Jun Ai
}

State Key Laboratory of Organ Failure Research, National Clinical Research Center of Kidney Disease, Division of Nephrology, Nanfang Hospital, Southern Medical University, Guangzhou, PR China

\section{Keywords}

Serum phosphorus controlled duration · Serum

phosphorus changed degree · Peritoneal dialysis · Overall

mortality · Peritoneal dialysis withdrawal

\begin{abstract}
Background: Serum phosphorus (SP) level is closely associated with overall mortality and cardiovascular events, while the role of SP controlled duration is not fully recognized. Here, we conducted a retrospective cohort study in our department to identify the relationship of SP controlled duration with clinical outcomes in patients undergoing peritoneal dialysis (PD). Methods: PD patients in our center from January 1, 2009, to June 30, 2019, were followed up at 2-month (the first year) or 5-month (the next follow-up period) intervals, and until death, until PD withdrawal, or until June 30, 2019. Data at each follow-up point were collected from their medical records. SP levels, changed degree of SP over baseline, and SP controlled duration were analyzed with overall mortality, PD withdrawal (including death,
\end{abstract}

Nirong Gong and Zhiwen Xiao contributed equally to this work. transferred to hemodialysis, and received renal transplantation), and combined endpoint (including death, acute heart failure, cardiovascular event, and stroke). Results: A total of 530 patients entered the analysis. Of them, 456 (86.0\%) had hyperphosphatemia before dialysis, and the SP levels decreased soon after dialysis. The degree of SP change over baseline was the maximum at the 3rd month after dialysis $(-31.0 \%)$, and lower degree was associated with higher overall mortality (hazard ratio [HR], 1.012; 95\% Cl, 1.004-1.020; $p=0.003)$. The median SP controlled duration was 13 (5-28) months, and longer duration was significantly associated with lower overall mortality $(\mathrm{HR}, 0.968 ; 95 \% \mathrm{Cl}, 0.956-0.981$; $p<0.001)$. After categorization, duration more than 12 months greatly improved overall mortality with a HR of 0.197 (0.082-0.458; $p<0.001$ vs. SP never controlled group) and $0.329(0.150-0.724 ; p=0.006$ vs. duration $<12$ months group). Longer SP controlled duration also improved PD withdrawal and combined endpoint. Conclusions: In summary, both degree and duration of SP control were tightly associated with overall mortality. We should control SP levels as early, as possible, and as long as we could.

(c) 2020 The Author(s)

Published by S. Karger AG, Basel

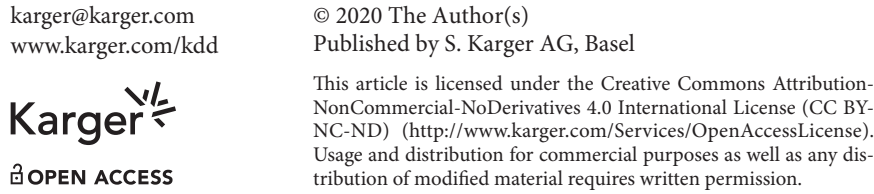

Dr. Jun Ai or Dr. Jing Nie

Division of Nephrology, Nanfang Hospital

1838 North Guangzhou Ave.

Guangzhou 510515 (PR China)

aij1980@163.com or jingnie2006@163.com 


\section{Introduction}

Peritoneal dialysis (PD) is a high quality and cost-effective dialysis modality of renal replacement treatment. About 7-40\% ESRD patients receive PD in the world [14]. The ratio is increasing in many countries, especially in China and East Asia [1-4]. About $40-60 \%$ of death in PD patients is associated with cardiovascular events [5-7]. Cardiovascular mortality is associated not only with conventional risk factors, such as age, hypertension, and diabetes mellitus, but also with vascular calcification and stiffness in ESRD patients [5-10].

Phosphorus metabolism disorder is a common complication of ESRD, which plays an important role in bone integrity, ectopic calcium deposition and calciphylaxis, vascular calcification, and myocardial hypertrophy [11-13]. Many studies had clarified the importance of hyperphosphatemia in all-cause mortality and cardiovascular events in patients with PD [14-17]. With government medical insurance covering [18], dialysis and phosphate binder use have been popularized recently, which greatly improved serum phosphorus (SP) management in China. Even though numerous international studies have declared that SP level is tightly associated with overall mortality and cardiovascular events [14-17], the role of SP controlled duration is not fully recognized. In order to identify the role of SP controlled duration in clinical outcomes, we conducted a retrospective cohort study involving 530 patients undergoing PD in our department.

\section{Subjects and Methods}

\section{Patients and Follow-Up}

This was a retrospective, single-center, cohort study approved by the Division of Nephrology, Nanfang Hospital, Southern Medical University. All the participants enrolled in this study have provided written informed consent (ethics number NFEC-2019-107, approved by the Research Ethics Committee of Nanfang Hospital). All patients undergoing PD for more than 3 months and aged 18 years or older were eligible for this study from January 1, 2009, to June 30, 2019. Patients who were not willing to join the study or have not had regular follow-up (never followed up after PD catheter implantation, or did not follow up once a year after dialysis) were excluded. All patients were followed up at 2-month (for the first year) or 5-month (for the next follow-up period) intervals, and until death, until PD withdrawal, or until June 30, 2019.

\section{Data Collection and Calculation}

Baseline data were collected on demographic characteristics (age, gender, and BMI), reasons for ESRD, medical histories (hypertension, diabetic mellitus, and hepatitis B virus infection), PD vintage, $\mathrm{PD}$ modality, and peritoneal equilibration test (PET) types (1 month after PD). Data over time of systolic blood pressure; diastolic blood pressure; left ventricular ejection fraction; dialysate glucose concentration; 24-h PD ultrafiltrate volume; 24-h urine volume; weekly total KT/V; serum levels of $\mathrm{Cr}$, albumin, and calcium; intact parathyroid hormone; and blood hemoglobin were collected at baseline (before PD, 0 month) and at $6,12,24,36,48$, 72,96 , and 120 months after PD. In order to evaluate the detailed information of SP, levels at 0 month and at 3, 6, 9, 12, 18, 24, 30, $36,48,72,96$, and 120 months after PD were collected and recorded. Phosphorus binder (PB) use was also recorded including the beginning time. Information on patients' medical history and medications was obtained from their medical records. Serum $\mathrm{Cr}$, albumin, calcium, and phosphate were measured using an automatic biochemical analyzer (AU480; Olympus, Tokyo, Japan) in our hospital. Serum parathyroid hormone levels were measured by chemiluminescence assay (D-68350; Roche Diagnostics, Indianapolis, IN, USA). Hemoglobin was tested by a routine blood test analyzer (XN9000; Sysmex, Kobe, Japan).

Conventional weekly total KT/V, and PET types were measured by standard methods $[19,20]$. We quantified some calculation formulas as follows:

Degree of SP change over baseline $(\%)=($ SP level at the followup point - baseline SP level $) \times 100 /$ baseline SP level.

Duration of SP control (months) $=$ PD vintage when patients reached hyperphosphatemia $(\mathrm{PH})$ - $\mathrm{PD}$ vintage when patients' SP decreased to $<1.78 \mathrm{mmol} / \mathrm{L}$ after PD (PN). If patients' SP never reached $<1.78 \mathrm{mmol} / \mathrm{L}, \mathrm{PH}=0$ and $\mathrm{SP}$ controlled duration $=0$, while if patients never reached hyperphosphatemia after dialysis, $\mathrm{PH}=$ actual PD vintage till the end of the follow-up. For example, for a given patient, if SP at 1 month after PD was normal and was abnormal at 9 months after $\mathrm{PD}, \mathrm{PN}=1, \mathrm{PH}=9$, and $\mathrm{SP}$ controlled duration of this patient was $8(9-1)$ months.

Dialysate glucose concentration (\%) $=\Sigma$ (glucose concentration $\times$ input volume)/total input volume. For example, if a patient is treated by continuous ambulatory peritoneal dialysis with $1.5 \%$ dialysate twice per day $+2.5 \%$ dialysate twice per day, the glucose concentration $=(1.5 \% \times 2 L \times 2+2.5 \% \times 2 L \times 2) / 8 L=2.0 \%$.

Mean arterial pressure $(\mathrm{MAP}, \mathrm{mmHg})=$ diastolic pressure + (systolic pressure - diastolic pressure)/3.

\section{Outcomes and Definitions}

The primary outcome was overall mortality. The secondary outcomes were PD withdrawal (including death, transfer to hemodialysis, and renal transplantation) and combined endpoint (including death, acute heart failure, cardiovascular diseases [CVDs], and stroke). All these events were based on the physician diagnosis from the medical records. Anuria was defined as $24 \mathrm{~h}$ urine volume $<100 \mathrm{~mL}$.

\section{Statistical Analyses}

Stata 15 software was used for the statistical analyses. The $t$ test, Kruskal-Wallis test, or $\chi^{2}$ test was used to compare continuous or categorical variables for baseline characteristics by with or without anuria. For an overall comparison of SP changes over time between groups, random-effects model analyses were performed with particular focus on the interaction between group and time. Unadjusted and adjusted (with age, sex, diabetic kidney disease [DKD] to ESRD, and the mean values of MAP, dialysate glucose concentration, urine volume, KT/V, serum albumin, and HGB during the 
first 12 months after dialysis) Cox regression analyses were performed to determine the relationships of SP level, degree of SP change over baseline, and duration of SP control with overall mortality. We categorized the duration of SP control into 5 groups and then performed Kaplan-Meier and Cox regression analyses on the created variables. Kaplan-Meier and Cox regression analyses were also performed to identify the relationships of SP controlled duration with PD withdrawal and combined endpoint, and the relationships of $\mathrm{PB}$ prescription with overall mortality, $\mathrm{PD}$ withdrawal, and combined endpoint.

Continuous data were expressed as mean \pm SD or median (interquartile range [IQR]), categorical data were expressed as number (proportion), and HR data were expressed as HR (95\% CI). A value of $p<0.05$ was considered to be statistically significant.

\section{Results}

\section{Cohort Description}

There were 682 ESRD patients undergoing PD from January 1, 2009, to June 30, 2019, in our department. After exclusion, 530 patients finally entered the analysis (Fig. 1). Of these patients, the mean age was $45.4 \pm 15.0$ years, $303(57.2 \%)$ patients were male, $84(15.9 \%)$ patients' ESRD cause was DKD, and the median PD vintage was 32 (15-54) months (Table 1). During the follow-up period, 78 (14.7\%) patients died and 221 (41.7\%) patients developed anuria. Anuric patients had higher baseline MAP, higher baseline serum phosphate and parathyroid hormone levels, higher initial dialysate glucose concentration and ultrafiltrate volume, longer $\mathrm{PD}$ vintage, lower initial urine volume, and lower baseline weekly total KT/V score. These patients also had higher overall mortality and a higher incidence of combined endpoint (Table 1).

\section{Changes in SP Levels over Time}

About 456 patients (86.0\%) had hyperphosphatemia before peritoneal dialysis. Of them, 69 (15.1\%) patients' SP levels never controlled to $<1.78 \mathrm{mmol} / \mathrm{L}$ during the follow-up period, and the remaining 387 (84.9\%) patients' SP levels decreased to $<1.78 \mathrm{mmol} / \mathrm{L} 1$ (1-1) month after dialysis. In all, 256 patients' phosphorus levels returned to hyperphosphatemia 9 (3-23) months after PD (Fig. 2a). The SP levels returned to hyperphosphatemia in 125 (48.8\%), 67 (26.2\%), 40 (15.6\%), and 24 (9.4\%) patients at the first, second, third, and more than 4 years after dialysis, respectively (Fig. 2b). We also calculated the change in degree of SP level over baseline. Interestingly, the degree of SP changes over baseline was the maximum at the $3 \mathrm{rd}$ month after dialysis $(-31.0 \%$ [ -43.8 to $-14.7 \%])$ and decreased later (degrees of SP change more than 36

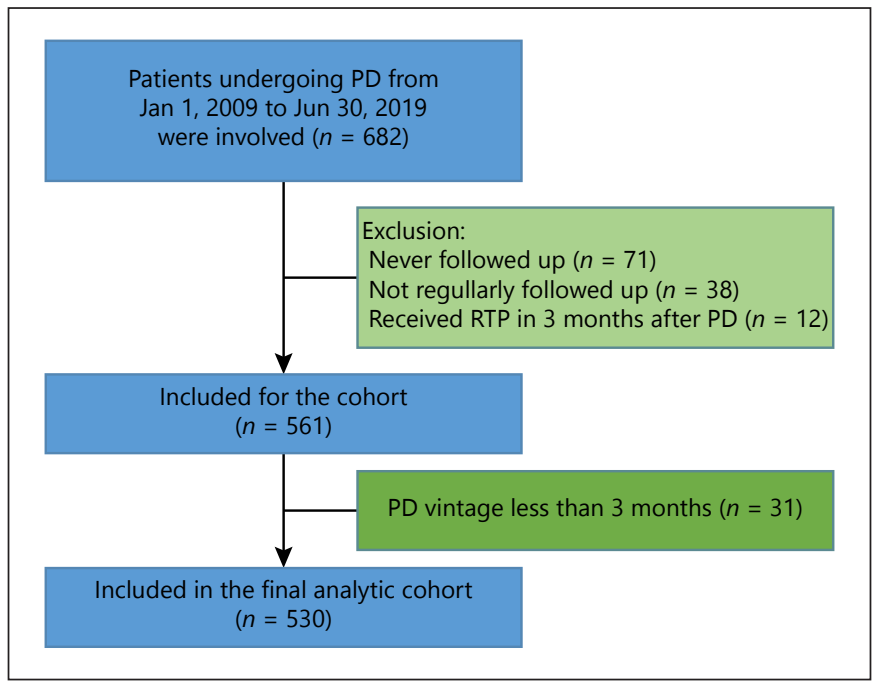

Fig. 1. Flowchart of patient selection into the study cohort. PD, peritoneal dialysis; RTP, renal transplantation.

months over baseline are not shown; see online suppl. Fig. 1a; for all online suppl. material, see www. karger.com/doi/10.1159/000507785). Details of changes in degrees in patients at the first, second, third, and more than 4 years after dialysis are shown in online suppl. Fig. $1 b$.

\section{Relationship between Overall Mortality and SP Situation}

Because the SP level is tightly associated with overall mortality [14-17], we next analyzed the relationships of overall mortality with SP levels and changes in degrees of SP level over baseline at each follow-up point (those more than 36 months are not shown). As shown in online suppl. Table 1, baseline SP level was not associated with overall mortality (HR, 0.764; 95\% CI, 0.521-1.119; $p=0.166$ ), while SP level at the 3rd month after dialysis (HR, 1.666; 95\% CI, $1.007-2.758 ; p=0.047$ ) was significantly associated with overall mortality after adjusted for demographics (age and sex), DKD to ESRD, and the mean values of MAP, dialysate glucose concentration, urine volume, weekly total KT/V, serum albumin, and HGB during the first 12 months after dialysis. SP levels at the other followup points did not show any significance with overall mortality. Interestingly, lower changed degree of the 3rd month's SP over baseline was independently associated with higher overall mortality (HR, 1.012; 95\% CI, 1.004$1.020 ; p=0.003)$. Degrees of SP change over baseline at the other follow-up points were not associated with overall mortality after adjusted Cox regression analysis. 
Table 1. Clinical characteristics of the study cohort

\begin{tabular}{|c|c|c|c|c|}
\hline General characteristic & $\begin{array}{l}\text { Overall } \\
(n=530)\end{array}$ & $\begin{array}{l}\text { Without anuria } \\
(n=309)\end{array}$ & $\begin{array}{l}\text { With anuria } \\
(n=221)\end{array}$ & $p$ value $^{\mathrm{a}}$ \\
\hline Age, years & $45.4 \pm 15.0$ & $44.6 \pm 14.2$ & $46.6 \pm 16.0$ & 0.139 \\
\hline MAP, mmHg & $107.8 \pm 13.8$ & $106.7 \pm 12.8$ & $109.4 \pm 15.1$ & 0.020 \\
\hline DKD to ESRD & $84(15.9)$ & $46(14.9)$ & $38(17.2)$ & 0.539 \\
\hline Serum ALB, g/L & $34.4 \pm 5.8$ & $35.0 \pm 5.3$ & $33.6 \pm 6.3$ & 0.006 \\
\hline Blood HGB, g/L & $87.9 \pm 22.3$ & $87.4 \pm 20.9$ & $88.8 \pm 24.2$ & 0.470 \\
\hline Serum calcium, mmol/L & $2.04 \pm 0.29$ & $2.05 \pm 0.29$ & $2.03 \pm 0.29$ & 0.412 \\
\hline Serum phosphate, $\mathrm{mmol} / \mathrm{L}$ & $2.35 \pm 0.64$ & $2.27 \pm 0.61$ & $2.46 \pm 0.68$ & 0.001 \\
\hline Serum PTH, pg/mL & $360.4 \pm 294.6$ & $332.7 \pm 211.3$ & $402.5 \pm 385.2$ & 0.009 \\
\hline Urine volume, $\mathrm{mL} / 24 \mathrm{~h}$ & $800(500-1,100)$ & $900(600-1,200)$ & $650(350-1,000)^{\mathrm{b}}$ & $<0.001$ \\
\hline KT/V score & $2.32 \pm 0.63$ & $2.40 \pm 0.71$ & $2.20 \pm 0.60$ & 0.001 \\
\hline \multicolumn{4}{|l|}{ PET type } & 0.167 \\
\hline Low, $n(\%)$ & $24(4.5)$ & $15(4.9)$ & $9(4.1)$ & - \\
\hline Low average, $n(\%)$ & $236(44.5)$ & $145(46.9)$ & $91(41.2)$ & - \\
\hline High average, $n(\%)$ & $233(44.0)$ & $128(41.4)$ & $105(47.5)$ & - \\
\hline High, $n(\%)$ & $37(7.0)$ & $21(6.8)$ & $16(7.2)$ & - \\
\hline $\mathrm{PD}$ vintage, months & $32(15-54)$ & $21(11-38)$ & $49(32-70)$ & $<0.001$ \\
\hline SP controlled duration, months & $13(5-28)$ & $11(6-23)$ & $17(4-32)$ & 0.202 \\
\hline \multicolumn{5}{|l|}{ Outcomes } \\
\hline \multicolumn{5}{|c|}{$\begin{array}{l}\text { Continuous variables are expressed as mean } \pm \mathrm{SD} \text { or median }(25 \text { th percentile- } 75 \text { th percentile). Categorical variables are expressed as } \\
n(\%) \text {. MAP, KT/V, and PET were calculated by formulas mentioned before. MAP, mean arterial pressure; DKD, diabetic kidney disease } \\
\text { PTH, parathyroid hormone; PD, peritoneal dialysis; CAPD, continuous ambulatory peritoneal dialysis; GLUC, glucose concentration } \\
\text { UF, ultrafiltrate; HD, hemodialysis; PET, peritoneal equilibration test; SP, serum phosphorus. }{ }^{\mathrm{a}} p \text { value for comparisons between with } \\
\text { and without RRF groups by } t \text { test, Kruskal-Wallis test, or } \chi^{2} \text { test for continuous and categorical variables, respectively. }{ }^{\text {b }} \text { Because without } \\
\text { RRF was defined as anuria (urine volume }<100 \mathrm{~mL} / 24 \mathrm{~h} \text { ) during the whole follow-up period, patients were not anuric at baseline. }\end{array}$} \\
\hline
\end{tabular}

We then calculated the SP controlled duration of every patient and analyzed the relationship with overall mortality. The median SP controlled duration was 13 (5-28) months (Table 1). As presented in Table 2, the SP controlled duration was significantly associated with incident of overall mortality (HR, 0.971 ; 95\% CI, 0.959-0.984; $p<0.001)$. After adjustment with clinical variables, longer duration still correlated to overall mortality (HR, 0.968; 95\% CI, $0.956-0.981 ; p<0.001)$. Converting the duration unit from months to years, the HR was 0.619 (0.512$0.748)$.

Serum Phosphorus Controlled Duration Associated with Mortality
We next categorized the duration into 5 groups, including SP never controlled group, SP controlled $<12$ months group, 12-23 months group, 24-35 months group, and $>35$ months group. As shown in Figure 3, patients whose SP never controlled had the maximum overall mortality $(24.6 \%)$, and the mortality decreased with longer SP controlled duration ( $p=0.046$ for trend). Patients with $<12$ months of SP controlled duration had similar overall mortality to the SP never controlled group (HR, 0.598; 95\% CI, 0.321-1.158; $p=0.120$ ). When the duration prolonged to more than 12 months, the overall 
Fig. 2. Changes in SP levels over time in PD patients. a Overall changes in SP levels over time. b Changes in SP levels over time in categorized SP controlled duration groups. SP controlled duration was categorized into SP never controlled group, SP controlled $<12$ months group, $12-23$ months group, 24-35 months group, and >35 months group. Patients' SP levels decreased soon after dialysis and returned to hyperphosphatemia later (a). SP levels returned to hyperphosphatemia in 125 (48.8\%), 67 (26.2\%), 40 (15.6\%), and 24 (9.4\%) patients at the first, second, third, and more than 4 years after dialysis (b). PD, peritoneal dialysis; SP, serum phosphorus; SD, standard deviation.
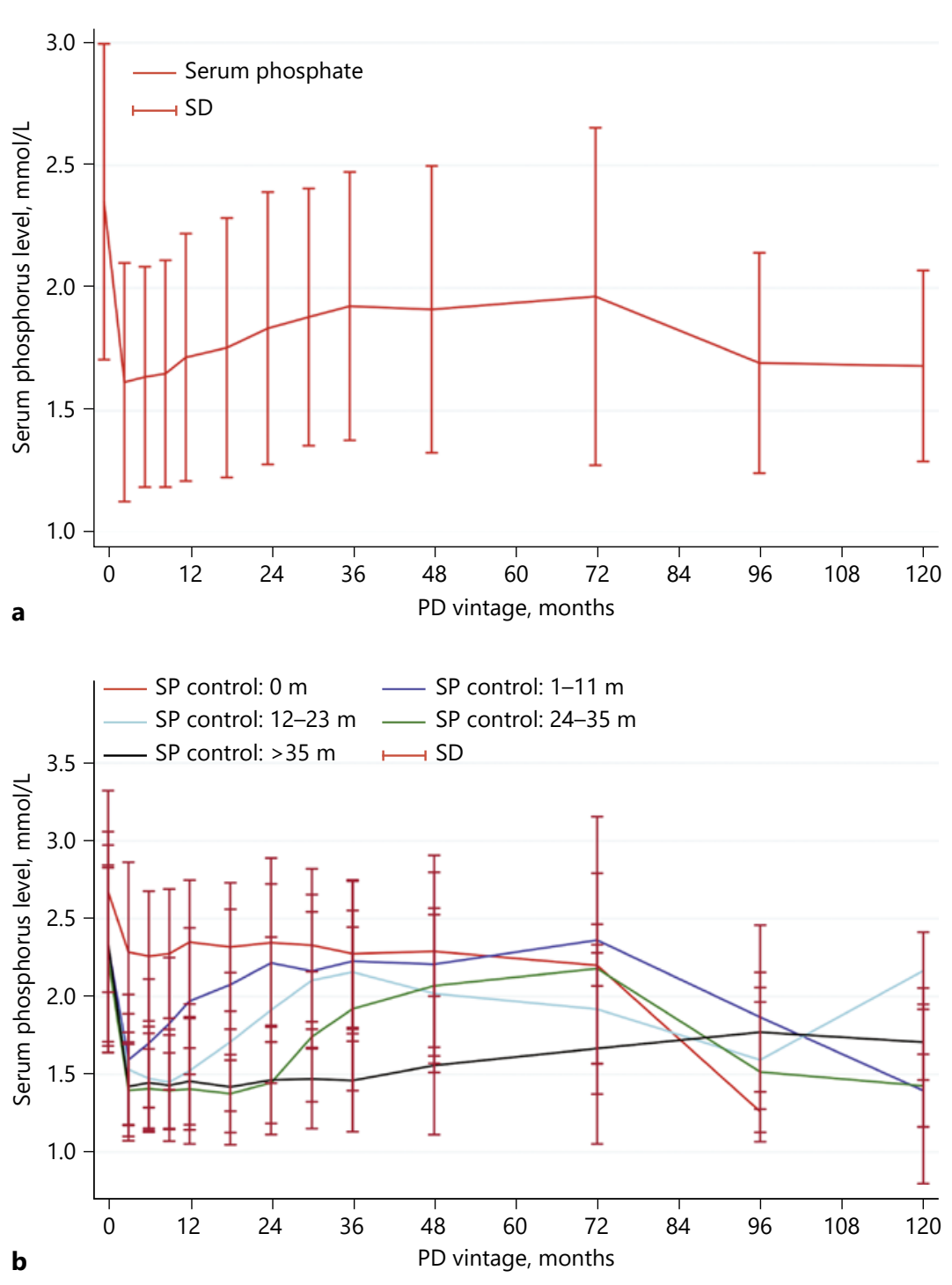

mortality greatly improved with HRs of 0.197 (0.082$0.458 ; p<0.001$ vs. SP never controlled group) and 0.329 $(0.150-0.724 ; p=0.006$ vs. SP controlled $<12$ months group) (Fig. 4; Table 3, and online suppl. Table 2). Even though the group presented no more benefits than the 12- to 23-month group, longer duration of more than 24 months still showed significant improvement for overall mortality (Fig. 4; Table 3, and online suppl. Table 2).

In order to confirm the relationship between SP controlled duration and overall mortality, we analyzed those patients who entered PD treatment from January 1, 2009, to June 30, 2017. For the 416 patients, we obtained similar results with lower censored data (online suppl. Table 3; Fig. 2).

\section{Relationship between Secondary Outcomes and SP Controlled Duration}

We also analyzed PD withdrawal and combined endpoint as secondary outcomes. During the follow-up period, 226 (42.7\%) patients had PD withdrawal (78 patients had died, 55 patients had transferred to hemodialysis, and 93 patients had received renal transplantation), and 196 (37.1\%) patients had reached combined endpoint (78 patients had died, 144 patients had acute heart failure, and 68 patients had CVD or stroke). As shown in Table 4, patients with longer SP controlled duration had lower incidence of PD withdrawal (HR, 0.964; 95\% CI, 0.954-0.973; $p<0.001$ ) and combined endpoint (HR, 
Table 2. Cox regression analysis for overall mortality

\begin{tabular}{|c|c|c|c|c|}
\hline \multirow[t]{2}{*}{ Variable } & \multicolumn{2}{|l|}{ Unadjusted } & \multicolumn{2}{|l|}{ Adjusted $^{\mathrm{a}}$} \\
\hline & HR (95\% CI) & $p$ value & HR (95\% CI) & $p$ value \\
\hline SP controlled duration, months & $0.971(0.959-0.984)$ & $<0.001$ & $0.968(0.956-0.981)$ & $<0.001$ \\
\hline SP controlled duration, years & $0.640(0.533-0.770)$ & $<0.001$ & $0.619(0.512-0.748)$ & $<0.001$ \\
\hline Age, years & $1.033(1.018-1.049)$ & $<0.001$ & $1.017(1.000-1.035)$ & 0.055 \\
\hline Male & $1.338(0.852-2.101)$ & 0.207 & $1.409(0.821-2.420)$ & 0.214 \\
\hline DKD to ESRD & $2.286(1.404-3.730)$ & 0.001 & $2.166(1.241-3.780)$ & 0.007 \\
\hline Mean MAP, mmHg & $0.963(0.943-0.984)$ & 0.001 & $0.963(0.942-0.986)$ & 0.001 \\
\hline Mean dialysate GLUC, \% & $4.702(1.702-9.747)$ & 0.002 & $1.632(0.577-4.617)$ & 0.356 \\
\hline Mean urine volume, $\mathrm{L}$ & $0.495(0.280-0.874)$ & 0.015 & $0.712(0.345-1.468)$ & 0.357 \\
\hline Mean serum ALB, g/L & $0.905(0.874-0.936)$ & $<0.001$ & $0.906(0.869-0.945)$ & $<0.001$ \\
\hline Mean HGB, g/L & $0.986(0.973-0.999)$ & 0.033 & $0.996(0.984-1.008)$ & 0.520 \\
\hline Mean KT/V & $0.483(0.299-0.781)$ & 0.003 & $0.983(0.622-1.555)$ & 0.943 \\
\hline
\end{tabular}

MAP and KT/V were calculated by formulas mentioned before. Mean MAP, dialysate GLUC, urine volume, serum ALB, HGB, and KT/V were the average values during the first 12 months after dialysis. HR, hazard ratio; CI, confidence interval; SP, serum phosphorus; DKD, diabetic kidney disease; ALB, albumin; HGB, hemoglobin; MAP, mean arterial pressure. ${ }^{a}$ Adjusted for demographics (age and sex), DKD to ESRD, and the mean values of MAP, dialysate GLUC, urine volume, serum ALB, HGB, and KT/V during the first 12 months after dialysis.

0.982; 95\% CI, 0.976-0.989; $p<0.001)$. Patients whose SP controlled duration was more than 12 months had significant lower incidence of PD withdrawal than the SP never controlled group (HR 0.342; 95\% CI, 0.214-0.546; $p<0.001$; online suppl. Fig. 3; Table 4) and SP controlled $<12$ months group (HR 0.491; 95\% CI, 0.333-0.723; $p<$ 0.001) (data not shown). Patients whose SP controlled duration was more than 35 months had lower incidence of combined endpoint (online suppl. Fig. 3; Table 5).

\section{$P B$ Use}

Finally, we analyzed PB use in these patients. As presented in online suppl. Table 6, 255 (48.6\%) patients had received $\mathrm{PB}$ treatment during the follow-up period, 97 (18.3\%) patients had received PB treatment before dialysis, the median beginning time was $5(0-24)$ months after dialysis, and $200(78.4 \%)$ patients had received non-calcium $\mathrm{PB}$. In the recent 4 years, more patients have received PB treatment ( 54.0 vs. $41.3 \%, p=0.004$ ) during the follow-up period, more patients have received $\mathrm{PB}$ treatment before dialysis $(24.1$ vs. $10.3 \%, p<0.001)$, and even more patients have received earlier (1 vs. 22.5 months after dialysis, $p<0.001)$ PB treatment. PB use was significantly associated with overall mortality (HR, 0.555; 95\% CI, 0.332-0.927; $p=0.025$ ) and PD withdrawal (HR, 0.523; 95\% CI, 0.391-0.698; $p<0.001$; online suppl. Fig. 4; Table 7).

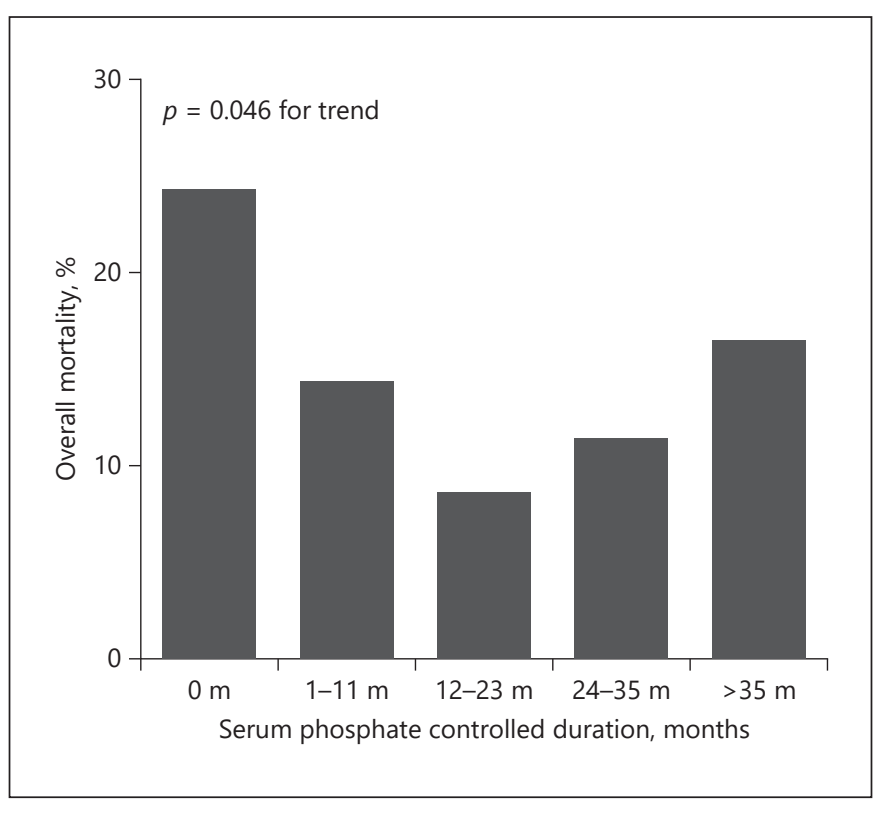

Fig. 3. Overall mortality in categorized SP controlled duration groups. SP controlled duration was categorized into SP never controlled group, SP controlled $<12$ months group, 12-23 months group, 24-35 months group, and $>35$ months group. The overall mortality was the maximum in patients whose SP never controlled and decreased with longer SP controlled duration. $p=0.046$ for trend. PD, peritoneal dialysis; SP, serum phosphorus. 
Fig. 4. Kaplan-Meier survival analyses of overall mortality. a Overall mortality in PD patients. b Overall mortality in categorized SP controlled duration groups. SP controlled duration was categorized into SP never controlled group, SP controlled $<12$ months group, 12-23 months group, 2435 months group, and $>35$ months group. Data are shown with censored data. ${ }^{*} p<$ 0.001 , SP controlled 12-23 months group versus SP never controlled group; ${ }^{* *} p=$ 0.006 , SP controlled $12-23$ months group versus SP controlled $<12$ months group. $\mathrm{PD}$, peritoneal dialysis; SP, serum phosphorus.

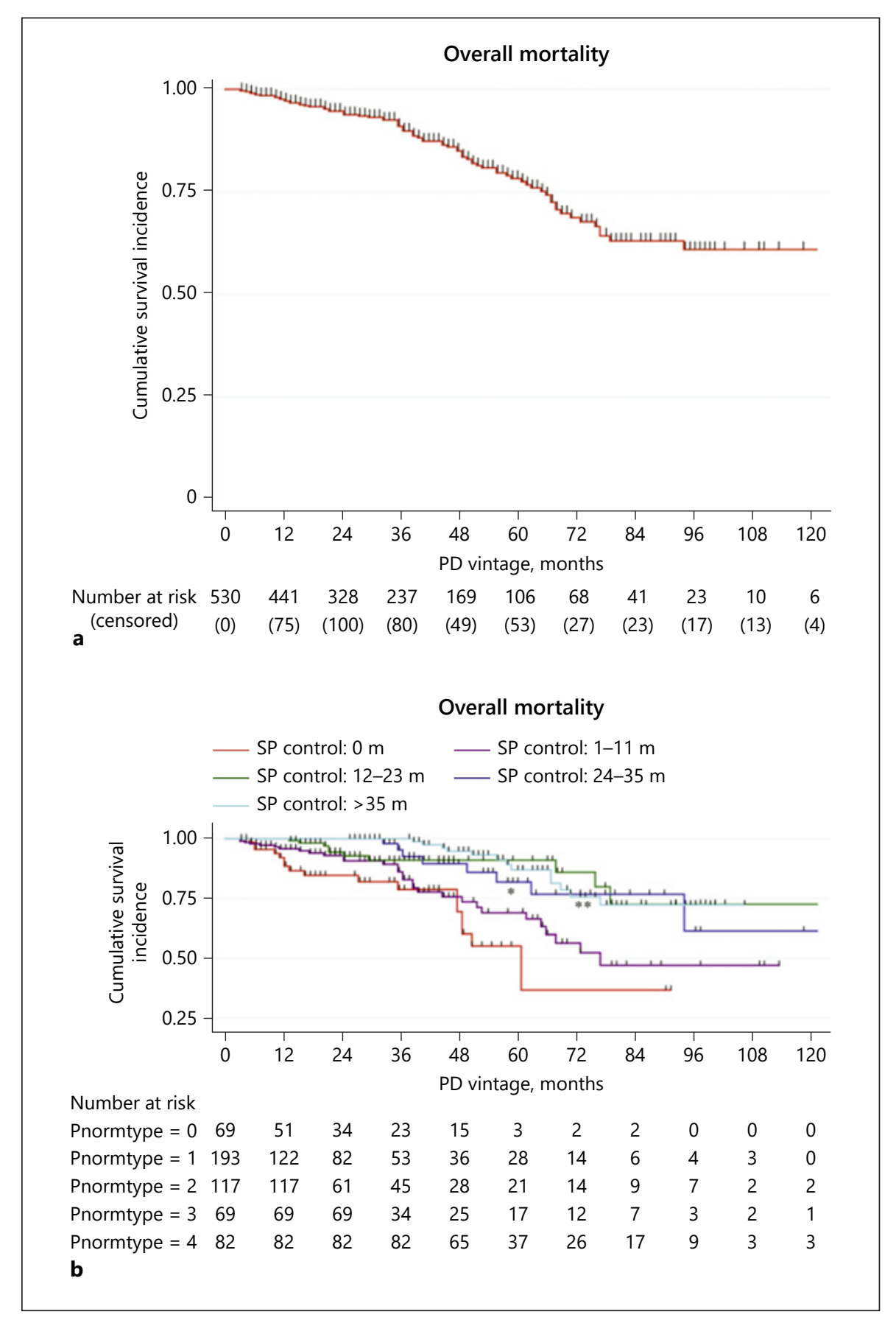

\section{Discussion}

In this retrospective cohort study, we identified that SP controlled duration was closely associated with overall mortality in patients undergoing PD. SP levels decreased to less than $1.78 \mathrm{mmol} / \mathrm{L}$ soon after $\mathrm{PD}$, and returned to hyperphosphatemia 9 months later. Longer duration of SP control was independently associated with lower overall mortality, with a $3.2 \%$ decrease of risk for every one more month of SP control and a 38.1\% decrease of risk for every one more year of SP control. Longer duration of SP control was also independently associated with lower PD withdrawal and combined endpoint. These data suggest that SP controlled duration is very important in PD patients. 
Table 3. Cox regression analysis for overall mortality with categorized SP controlled duration

\begin{tabular}{|c|c|c|c|c|}
\hline \multirow[t]{2}{*}{ SP controlled duration } & \multicolumn{2}{|l|}{ Unadjusted } & \multicolumn{2}{|l|}{ Adjusted $^{\mathrm{a}}$} \\
\hline & HR (95\% CI) & $p$ value & HR (95\% CI) & $p$ value \\
\hline 0 month & 1 (refer) & - & 1 (refer) & - \\
\hline $1-11$ months & $0.592(0.324-1.080)$ & 0.087 & $0.598(0.321-1.158)$ & 0.120 \\
\hline $12-23$ months & $0.228(0.103-0.501)$ & $<0.001$ & $0.197(0.082-0.458)$ & $<0.001$ \\
\hline 24-35 months & $0.231(0.099-0.540)$ & 0.001 & $0.279(0.114-0.683)$ & 0.005 \\
\hline$>35$ months & $0.193(0.092-0.403)$ & $<0.001$ & $0.143(0.064-0.331)$ & $<0.001$ \\
\hline
\end{tabular}

MAP and KT/V were calculated by formulas mentioned before. SP, serum phosphorus; HR, hazard ratio; CI, confidence interval; DKD, diabetic kidney disease; MAP, mean arterial pressure; GLUC, glucose concentration; ALB, albumin; HGB, hemoglobin. ${ }^{a}$ Adjusted for demographics (age and sex), DKD to ESRD, and the mean values of MAP, dialysate GLUC, urine volume, serum ALB, HGB, and KT/V during the first 12 months after dialysis.

Table 4. Cox regression analysis for PD withdrawal and combined endpoint with SP controlled duration

\begin{tabular}{|c|c|c|c|c|}
\hline \multirow[t]{2}{*}{ Variable } & \multicolumn{2}{|l|}{ PD withdrawal } & \multicolumn{2}{|l|}{ Combined endpoint } \\
\hline & HR (95\% CI) & $p$ value $^{\mathrm{a}}$ & HR (95\% CI) & $p$ value $^{\mathrm{b}}$ \\
\hline SP controlled duration, months & $0.964(0.954-0.973)$ & $<0.001$ & $0.982(0.976-0.989)$ & $<0.001$ \\
\hline SP controlled duration, years & $0.571(0.499-0.652)$ & $<0.001$ & $0.758(0.678-0.847)$ & $<0.001$ \\
\hline
\end{tabular}

KT/V was calculated by formulas mentioned before. SP, serum phosphorus; HR, hazard ratio; CI, confidence interval; GLUC, glucose concentration; ALB, albumin; DKD, diabetic kidney disease. ${ }^{a}$ Adjusted for demographics (age and sex) and the mean values of dialysate GLUC, urine volume, serum Cr, HGB, and KT/V during the first 12 months after dialysis. ${ }^{\mathrm{b}}$ Adjusted for demographics (age and sex), DKD to ESRD, and the mean values of dialysate GLUC, urine volume, serum ALB, HGB, and KT/V during the first 12 months after dialysis.

Large international studies have shown that elevated SP levels (hyperphosphatemia) are linked with a number of serious clinical complications, including vascular calcification $[11,12]$ and left ventricular hypertrophy [13], as well as increased all-cause mortality and PD withdrawal in patients undergoing dialysis [5-10, 14-17]. In the Dialysis Outcomes and Practice Patterns Study (DOPPS), SP levels decreased over time in all countries, and higher SP levels were associated with greater all-cause mortality risk in both baseline and time-dependent models $[14,15]$. In our center, most patients (86.0\%) had hyperphosphatemia at baseline, while few patients $(<1 \%)$ had hypophosphatemia $(<0.65 \mathrm{mmol} / \mathrm{L})$, which is not that similar to previous reports. Furthermore, baseline SP levels were not associated with overall mortality. In China, general medical examination has not been popularized till now, and most CKD patients could not be diagnosed or receive proper treatment including SP management until the last stage, which might be the reason why so many patients had hyperphosphatemia at baseline. And because of the very high incidence of hyperphosphatemia before dialysis, baseline SP levels did not correlate to overall mortality in ESRD patients. Earlier detection and management of hyperphosphatemia in CKD patients is an elephant in the room in China.

With the application of " $3 \mathrm{D}$ " treatment (diet control, dialysis, and drug use) [21-24], about $87.0 \%$ patients' SP levels decreased to $<1.78 \mathrm{mmol} / \mathrm{L}$ soon after dialysis, with a degree of $-31 \%$ over baseline, and increased again later, which is similar to previous reports [14-17]. Only $13 \%$ patients' SP levels never controlled to normal. Of note, the mean SP level of the 3rd month was the lowest during the follow-up period, and the degree of SP change at this point over baseline was the highest. Interestingly, only the 3rd month's SP level and the changed degree kept the significance with overall mortality after adjusted analysis, while those in other follow-up points did not show any significance. These data suggested that early SP level and changed degree after treatment might present more relationships with overall mortality in patients undergoing 
PD. Early and effective management of SP could practicably improve the survival rates of PD patients.

It is well known that SP level plays a very important role in ESRD patients $[5-10,25,26]$, while the role of duration of SP control is still not fully recognized. After calculating the SP controlled duration for every patient, we found that longer duration did correlate to lower overall mortality, with a 3.2 or $38.1 \%$ decrease of risk for every one more month or year of SP control. After categorization, patients with $<12$ months' duration had a similar overall mortality to those whose SP never controlled. When the duration prolonged to more than 12 months, the overall mortality greatly improved. Even though this presented no more benefits than the 12-23 months group, a longer duration of more than 24 months still showed significant improvement of overall mortality. Patients with longer SP controlled duration also had lower incidence of PD withdrawal and combined endpoint, including death, acute heart failure, CVD, and stroke. These data strongly suggested that longer duration of SP control might be even more important for PD patients, and we should pay adequate attention on prolonging the SP controlled duration.

Since a lower SP level of $<0.65 \mathrm{mmol} / \mathrm{L}$ was also associated with poorer outcomes in ESRD patients [14-16], we also analyzed patients whose SP level was $<0.65 \mathrm{mmol} / \mathrm{L}$. Unfortunately, we could not really identify the relationship between lower SP level and mortality because only $3(0.6 \%)$ patients' SP level reached $<0.65 \mathrm{mmol} / \mathrm{L}$ during the whole follow-up period. This might be because of the very high baseline hyperphosphatemia incidence or sample size.

With the government medical insurance covering, in recent years, more and more ESRD patients have received dialysis and phosphate binder treatment recently, improving SP management and prolonging the controlled duration. In the recent 4 years, more patients have received PD (307 vs. 223 ) and PB (54.0 vs. $41.3 \%)$, more patients have received $\mathrm{PB}$ at baseline (24.1 vs. $10.3 \%$ ), and patients had received $\mathrm{PB}$ earlier. This is an advantage of the medical insurance covering in recent years. Of note, PB use was significantly associated with overall mortality and PD withdrawal, which is similar to the previous report [27-29]. Because water and sodium retention might be a major reason for acute heart failure in PD patients, PB use seemed to not correlate with combined endpoints including acute heart failure. When combining only death, CVD, and CBD, PB use showed significant association (data not shown).

Besides hyperphosphatemia, hypertension and anemia are proved to be important risk factors for poor out- comes in PD patients. Most patients had anemia (84\%) and hypertension $(72 \%)$ at baseline, which might be the reason why baseline blood pressure or HGB did not correlate to overall mortality in this cohort. Baseline serum albumin showed some relationship with overall mortality (HR, 1.565; 95\% CI, 0.984-2.487; $p=0.058$, data were not shown). Interestingly, the mean values of blood pressure, blood HGB, and serum albumin did correlate to overall mortality and PD withdrawal incidence. We should pay attention to management of blood pressure, blood HGB, and nutrition because the mean values did correlate to overall mortality and combined endpoint.

This study had several limitations. First, this is a retrospective cohort study, and all the data were obtained from their medical records and the laboratory examination system of Nanfang Hospital. A prospective study should be conducted to confirm the relationship between SP controlled duration and clinical outcomes. Second, the relationship between lower SP level and outcomes has not been analyzed because fewer patients had SP level $<0.65$ $\mathrm{mmol} / \mathrm{L}$ during the follow-up period in this population. A larger sample size is needed to ensure the relationship.

In conclusion, this study provides a unique description of SP changes during several years in our center and identifies the relationships with overall mortality. For serum phosphorus, both the controlled degree over baseline and controlled duration are tightly associated with overall mortality. We should control SP levels as early as we could, as possible as we could, and as long as we could.

\section{Acknowledgements}

We thank Dr. Jianping Jiang and Dr. Hongfa Liu in our department for their important works in PD patients' general follow-up and management in our department, and we also thank all the nurses at our center for the patients' follow-up and the detailed medical records.

\section{Statement of Ethics}

All the participants enrolled in this study have provided written informed consent. The ethics number is NFEC-2019-107, which was approved by the Research Ethics Committee of Nanfang Hospital.

\section{Conflict of Interest Statement}

All authors have nothing to disclose with regard to commercial support. 


\section{Funding Sources}

This work was supported by the Nature and Science Foundation of China (No. 81770765) and Outstanding Youths Development Scheme of Nanfang Hospital, Southern Medical University (No. 2017J013) to Dr. Jun Ai.

\section{Author Contributions}

Nirong Gong and Zhiwen Xiao did the major works, including patient recruitment, data recording and checking, and data entry. Fen Zhang, Xiaohong Zhong, Yanfang He, Zhixiu Yi, Yanhong Lin, Cong Yang, and Dan Tang did some works in data recording, checking, and entry. Jun Ai designed the retrospective cohort study, finished the statistical analysis, and wrote the manuscript. Jing Nie helped in the study design and manuscript writing.

\section{References}

1 Mehrotra R, Devuyst O, Davies SJ, Johnson DW. The current state of peritoneal dialysis. J Am Soc Nephrol. 2016;27(11):3238-52.

2 Blake PG. Peritoneal dialysis in Asia: an external perspective. Perit Dial Int. 2002;22(2): 258-64.

3 National Institute of Health. US renal data system 2015 annual data report. Epidemiology of kidney disease in the United States. Chapter 13: international comparisons. Am J Kidney Dis. 2016;67(Suppl 1):S291-334.

4 Li PK, Chow KM, Van de Luijtgaarden MW, Johnson DW, Jager KJ, Mehrotra R, et al. Changes in the worldwide epidemiology of peritoneal dialysis. Nat Rev Nephrol. 2017; 13(2):90-103.

5 Krediet RT, Balafa O. Cardiovascular risk in the peritoneal dialysis patient. Nat Rev Nephrol. 2010;6(8):451-60.

6 Johnson DW, Dent H, Hawley CM, McDonald SP, Rosman JB, Brown FG, et al. Association of dialysis modality and cardiovascular mortality in incident dialysis patients. Clin J Am Soc Nephrol. 2009;4(10):1620-8.

7 Ladhani M, Craig JC, Irving M, Clayton PA, Wong G. Obesity and the risk of cardiovascular and all-cause mortality in chronic kidney disease: a systematic review and meta-analysis. Nephrol Dial Transplant. 2017;32(3):439-505.

8 Shaman AM, Kowalski SR. Hyperphosphatemia Management in Patients with Chronic Kidney Disease. Saudi Pharm J. 2016;24(4): 494-505.

9 Noordzij M, Cranenburg EM, Engelsman LF, Hermans MM, Boeschoten EW, Brandenburg VM, et al. Progression of aortic calcification is associated with disorders of mineral metabolism and mortality in chronic dialysis patients. Nephrol Dial Transplant. 2011; 26(5):1662-9.

10 Sipahioglu MH, Kucuk H, Unal A, Kaya MG, Oguz F, Tokgoz B, et al. Impact of arterial stiffness on adverse cardiovascular outcomes and mortality in peritoneal dialysis patients. Perit Dial Int. 2012;32(1):73-80.

11 Giachelli CM. Vascular calcification: in vitro evidence for the role of inorganic phosphate. J Am Soc Nephrol. 2003;14(9 Suppl 4):S300-4.
12 Carrillo-López N, Panizo S, Alonso-Montes C, Martínez-Arias L, Avello N, Sosa P, et al. High-serum phosphate and parathyroid hormone distinctly regulate bone loss and vascular calcification in experimental chronic kidney disease. Nephrol Dial Transplant. 2019;6: 934-41.

13 Neves KR, Graciolli FG, dos Reis LM, Pasqualucci CA, Moysés RM, Jorgetti V. Adverse effects of hyperphosphatemia on myocardial hypertrophy, renal function, and bone in rats with renal failure. Kidney Int. 2004;66(6): 2237-44.

14 Tentori F, Blayney MJ, Albert JM, Gillespie BW, Kerr PG, Bommer J, et al. Mortality risk for dialysis patients with different levels of serum calcium, phosphorus, and PTH: the Dialysis Outcomes and Practice Patterns Study (DOPPS). Am J Kidney Dis. 2008;52(3):51930.

15 Lopes AA, Tong L, Thumma J, Li Y, Fuller DS, Morgenstern $\mathrm{H}$, et al. Phosphate binder use and mortality among hemodialysis patients in the Dialysis Outcomes and Practice Patterns Study (DOPPS): evaluation of possible confounding by nutritional status. Am J Kidney Dis. 2012;60(1):90-101.

16 Floege J, Kim J, Ireland E, Chazot C, Drueke T, de Francisco A, et al. Serum iPTH, calcium and phosphate, and the risk of mortality in a European haemodialysis population. Nephrol Dial Transplant. 2011;26(6):1948-55.

17 Shang D, Xie Q, Ge X, Yan H, Tian J, Kuang $\mathrm{D}$, et al. Hyperphosphatemia as an independent risk factor for coronary artery calcification progression in peritoneal dialysis patients. BMC Nephrol. 2015;16:107.

18 Wang Z, Zhang Y, Xiong F, Li H, Ding Y, Gao $\mathrm{Y}$, et al. Association between medical insurance type and survival in patients undergoing peritoneal dialysis. BMC Nephrol. 2015;16: 33.

19 Mehrotra R, Ravel V, Streja E, Kuttykrishnan S, Adams SV, Katz R, et al. Peritoneal equilibration test and patient outcomes. Clin J Am Soc Nephrol. 2015;10(11):1990-2001.

20 National Kidney Foundation Dialysis Outcomes Quality International Peritoneal Ade- quacy Work Group. NKF-DOQI clinical practice guidelines for peritoneal dialysis adequacy. National Kidney Foundation. Am J Kidney Dis. 1997;30(3 Suppl 2):S67-136.

21 National Kidney Foundation. K/DOQI clinical practice guidelines for bone metabolism and disease in chronic kidney disease. Am J Kidney Dis. 2003;42(4 Suppl 3):S1-201.

22 Lynch KE, Lynch R, Curhan GC, Brunelli SM. Prescribed dietary phosphate restriction and survival among hemodialysis patients. Clin J Am Soc Nephrol. 2011;6(3):620-9.

23 Kuhlmann M. Phosphate elimination in modalities of hemodialysis and peritoneal dialysis. Blood Purif. 2010;29(2):137-44.

24 Floege J, Covic AC, Ketteler M, Mann J, Rastogi A, Spinowitz B, et al. One-year efficacy and safety of the iron-based phosphate binder sucroferric oxyhydroxide in patients on peritoneal dialysis. Nephrol Dial Transplant. 2017;32(11):1918-26.

25 Rivara MB, Ravel V, Kalantar-Zadeh K, Streja E, Lau WL, Nissenson AR, et al. Uncorrected and albumin-corrected calcium, phosphorus, and mortality in patients undergoing maintenance dialysis. J Am Soc Nephrol. 2015;26(7): 1671-81.

26 Wang AY. Cardiovascular risk factors in peritoneal dialysis patients revisited. Perit Dial Int. 2007;27(Suppl 2):S223-7.

27 Evenepoel P, Selgas R, Caputo F, Foggensteiner L, Heaf JG, Ortiz A, et al. Efficacy and safety of sevelamer hydrochloride and calcium acetate in patients on peritoneal dialysis. Nephrol Dial Transplant. 2009;24(1):278-85.

28 Katopodis KP, Andrikos EK, Gouva CD, Bairaktari ET, Nikolopoulos PM, Takouli LK, et al. Sevelamer hydrochloride versus aluminum hydroxide: effect on serum phosphorus and lipids in CAPD patients. Perit Dial Int. 2006;26(3):320-7.

29 Joy MS, Finn WF; LAM-302 Study Group Randomized, double-blind, placebo-controlled, dose-titration, phase III study assessing the efficacy and tolerability of lanthanum carbonate: a new phosphate binder for the treatment of hyperphosphatemia. Am J Kidney Dis. 2003;42(1):96-107.
Serum Phosphorus Controlled Duration Associated with Mortality 